\title{
Classic Psychedelic Coadministration with Lithium, but Not Lamotrigine, is Associated with Seizures: An Analysis of Online Psychedelic Experience Reports
}

\author{
Authors \\ Affiliations \\ 1 Department of Psychiatry and Behavioral Sciences, Johns \\ Hopkins University School of Medicine, Baltimore, MD, USA \\ 2 Erowid Center, Grass Valley, CA, USA \\ 3 Department of Neuroscience, Johns Hopkins University \\ School of Medicine, USA
}

Sandeep M. Nayak¹, Natalie Gukasyan¹, Frederick S. Barrett ${ }^{1}$, Earth Erowid ${ }^{2}$, Fire Erowid ${ }^{2}$, Roland R. Griffiths ${ }^{1,3}$

Key words

psychedelic, lithium, hallucinogen, harm, risks

received 28.02.2021

revised 11.05 .2021

accepted 25.05.2021

published online $\quad 04.08 .2021$

Bibliography

Pharmacopsychiatry 2021; 54: 240-245

DOI 10.1055/a-1524-2794

ISSN $0176-3679$

(c) 2021. Thieme. All rights reserved.

Georg Thieme Verlag KG, Rüdigerstraße 14,

70469 Stuttgart, Germany

Correspondence

Sandeep M. Nayak, MD

Center for Psychedelic and Consciousness Research

Johns Hopkins University School of Medicine

5510 Nathan Shock Drive

21224 Baltimore MD

USA

smn@jhmi.edu $\circledast_{\text {https://doi.org/10.1055/a-1524-2794. }}^{\text {Supplementary Material is available under }}$

\section{ABSTRACT}

Introduction Psychedelics show promise in treating unipolar depression, though patients with bipolar disorder have been excluded from recent psychedelic trials. There is limited information on the use of classic psychedelics (e. g., LSD or psilocybin) in individuals using mood stabilizers to treat bipolar disorder. This is important to know, as individuals with bipolar depression may attempt to treat themselves with psychedelics while on a mood stabilizer, particularly given enthusiastic media reports of the efficacy of psilocybin for depression.

Methods This study analyzed reports of classic psychedelics administered with mood stabilizers from 3 websites (Erowid. org, Shroomery.org, and Reddit.com).

Results Strikingly, $47 \%$ of 62 lithium plus psychedelic reports involved seizures, and an additional $18 \%$ resulted in bad trips while none of 34 lamotrigine reports did. Further, $39 \%$ of lithium reports involved medical attention. Most of the lamotrigine reports (65\%) but few ( $8 \%$ ) of the lithium reports were judged to not affect the psychedelic experience.

Discussion Although further research is needed, we provisionally conclude that psychedelic use may pose a significant seizure risk for patients on lithium.

\section{Introduction}

After more than 6 decades of research into and popular use of classic psychedelics (defined here as a class of drugs that produce a unique profile of subjective effects and have a principal site of action at the $5 \mathrm{HT}_{2 \mathrm{~A}}$ receptor, such as LSD or psilocybin), recent promising trials investigating the effects of psilocybin therapy for the treatment of depression [1,2], anxiety and depression in cancer patients [3,4], tobacco smoking [5], and alcohol use disorder [6] have inspired additional medical, commercial, and public interest in using psychedelics as treatments for psychiatric disorders. Interest has also grown in understanding whether psilocybin therapy may be effective for a wider range of indications [7-9].
Depressive episodes in individuals with bipolar disorder ("bipolar depression") in particular have been discussed as targets of interest, with at least one study planned in patients with bipolar II disorder (Clinicaltrials.gov identifier NCT04433845). However, the risk of adverse events in this patient population merits special consideration in light of the possibility of precipitating mania and concerns about possible interactions with other drugs commonly used to treat bipolar disorder.

Bipolar disorder is a chronic condition characterized by episodes of mania or hypomania and predominantly depressive episodes [10]. Pharmacologic treatment of bipolar disorder can be challenging due to the need to address alternating extremes of mood [11]. 
Treatment usually includes "mood stabilizers" such as lithium, certain anti-epileptic drugs, and anti-psychotics. Lithium remains the gold-standard treatment with particular efficacy for relapse prevention and treatment of acute mania $[12,13]$. However, treatment options specifically targeting depressive episodes in bipolar disorder are limited.

Modern psychedelic trials typically exclude people with bipolar disorder [14]. This was considered to be a prudent safety measure, given the potential for precipitating psychosis or mania in patients with a personal or family history of schizophrenia or bipolar spectrum disorders [15], but it also leaves open the question of whether psychedelic drugs may be effective in treating patients with bipolar depression. The combination of promising evidence for psilocybin in treating unipolar depression, the fact that bipolar depression is difficult to treat, the lack of clear information on problematic drug interactions, the increasing availability of psilocybin mushrooms, and the extensive positive media coverage of anecdotal reports suggesting therapeutic benefits of psilocybin in depression may lead individuals with bipolar disorder to take psychedelic drugs in addition to continuing their existing medications.

There is very little information concerning the effects of concurrent psychedelics and mood stabilizers. An online survey noted 2 subjects who reported taking LSD while on lithium $(600 \mathrm{mg}$ and $1000 \mathrm{mg}$ ) and described intensified subjective effects from LSD [16].

Here we report on anecdotal descriptions of reactions to the use of psychedelic drugs when taken with a concurrent mood stabilizer. Data were drawn from a systematic search of self-reported experiences posted or published in public online forums focused on psychedelic drugs. While this method cannot conclusively determine safety, it could establish some signal for risk in the potential for harmful interactions between psychedelic and typical therapeutic drugs.

\section{Methods}

\section{Data collection}

Three websites were used as data sources-Erowid.org, Shroomery. org, and Reddit.com. These are publicly accessible sites and online communities that include user-created and user-driven content in which people can anonymously share information related to psychedelic drug use. These online communities frequently receive "trip reports" (henceforth experience reports), or descriptions, often quite detailed, of psychedelic drug experiences. Though mostly anonymous, individuals typically post under a pseudonym. Experience reports on these websites are organized in a fashion that is amenable to automated collection and analysis of these reports (see individual descriptions below). For this paper, reports from each internet site were filtered for duplicate usernames to eliminate double-reporting on the same individual to the extent possible.

Most experience reports did not include demographic information such as age, prior psychedelic use, psychiatric and medical history, etc. in any systematic way, so these were not collected.

Experience reports were searched for a series of terms related to the use of mood stabilizers in combination with psychedelics. Antipsychotics and antidepressants were not separately queried, but the presence of an antipsychotic or antidepressant (among other psychoactive drugs) was coded in all selected experience reports at a later stage (see Coding of Reports below). Mood stabilizer search terms included: lithium, lamotrigine, valproic acid, carbamazepine, and oxcarbazepine, including generic and trade names and different chemical names (e. g., valproate, Depakote, etc.) Because good quality empirical laboratory studies have been published involving coadministration of haloperidol and risperidone with psilocybin, antipsychotics were not included in the search of the current, lower-quality data [17]. Psychedelic search terms included LSD, psilocybin mushrooms, DMT, mescaline, peyote, San Pedro, and ayahuasca, all of which are $5-\mathrm{HT}_{2 \mathrm{~A}}$ receptor agonists commonly used in recreational settings.

\section{Erowid}

Erowid is a curated online resource founded in 1995 for information related to psychoactive drugs, which also contains sections for user-generated and submitted content including experience reports for each drug and drug class that is covered on the website. Erowid's reports are tagged before publication with the drugs described in each report. Erowid has an application programming interface (API)-a purpose-built programming interface typically used to allow programmers to gain custom access to a data source or computing infrastructure. An API provided by Erowid was accessed via a custom python script on August 7, 2020, and used to identify and collect experience reports for the current analysis.

\section{Shroomery}

The Shroomery is an online community related to psychedelic drug use, nominally focused on psilocybin mushroom use but containing information posted by users for a wide range of psychedelic substances. The Shroomery has a specific section of its website dedicated to experience reports. Experience report entries on Shroomery.org were accessed on August 21, 2020, with a custom programming script written using $R$ statistical software [18] using the packages rvest [19] and RSelenium [20]. These packages allow for "web scraping," which is a systematic search and accessing of all of the contents of a given website without the need for an API.

In addition to specific experience reports, Shroomery.com also has a wide array of additional forums with posts that could have relevance. These were not searched as those posts would require excessive manual coding to extract the relevant data and are far less likely to include codable and relevant data than the specific "trip reports" section of the community.

\section{Reddit}

Reddit is one of the largest collections of online communities in the world, with innumerable "subreddits" or sections dedicated to particular topics. Reddit was chosen as a third source due to ease of access to data through an API. Posts on the subreddits r/Drugs, $r /$ Shrooms, r/Psychonaut, r/TripReports, r/Psychedelics, r/LSD, r/ Acid, and r/Epilepsy were accessed on August 22, 2020, with a script written in the python coding language [21] and using the Python Reddit API Wrapper (PRAW), a python package designed to interface with Reddit's API [22]. Search terms were similar to the above. 


\section{Coding of reports}

All experience reports (including comments, in the case of Reddit posts) that contained both a mood stabilizer search term and a psychedelic search term were collected and then manually coded by a research assistant and an author (SMN) for the presence of direct first-person or witnessed second-person reports of the experience encountered when a psychedelic was consumed by an individual who was also taking a mood stabilizer. This step was mainly relevant for Reddit as experience reports from Erowid and the Shroomery were already coded by drug pre-selected to involve accounts of consumption. In contrast, Reddit posts frequently included questions and comments without specific accounts of drug use.

Those experience reports that contained such a first- or secondhand report were then coded for the presence or absence of the mention of other psychiatric medications and psychoactive drugs (including caffeine, cannabinoids, benzodiazepines, and antipsychotics, stimulants, etc.), the reported effect, and whether or not a microdose of a psychedelic had been consumed. If the dose was not mentioned, it was assumed that the reported experience occurred in the context of a typical psychoactive dose (i. e., not a microdose). Experience reports that included both lithium and lamotrigine were removed ( 2 from Erowid, 0 from Shroomery, and 2 from Reddit), to separately compare reports of these 2 mood stabilizers taken with psychedelics. Three Reddit posts with duplicate content were identified and removed.

Experience reports involving "microdoses" of psychedelics-very low doses with minimal subjective effects-were not included. Likewise, very small doses of lithium (such as less than $30 \mathrm{mg}$, as is sometimes taken in the form of lithium orotate over the counter) were also not included due to less likelihood of physiological effects with low doses and the different clinical population that might take these.

Effects were coded in 6 mutually exclusive categories based on the author's description and interpretation of the effect of the concomitant mood-stabilizing medication on the psychedelic drug effect. 1) "seizure" was coded if the report indicated a seizure; 2 ) "bad trip" was coded if there was an explicit description of an overall negatively valenced or noxious experience excluding seizure; 3 ) "intensified psychedelic effects" was coded if the medication intensified the psychedelic effects excluding reports coded as seizures or bad trips; 4) "decreased psychedelic effect" was coded if the medication reduced the subjective psychedelic effect excluding reports coded as no psychedelic effect; 5) "no psychedelic effect" was coded if the medication completely prevented a subjective psychedelic effect, and 6) "neutral" was coded if there was either no particular mention of an effect or an explicit mention of no effect on the psychedelic experience. SMN manually coded these reports, which were then reviewed by NG, with discrepancies resolved by discussion.

\section{Results}

\section{Experience Reports}

All reports from Erowid and the Shroomery that met search criteria also met criteria for analysis as they were already selected to in- clude an account of a drug experience and organized by drugs consumed. In contrast, Reddit required a great degree of manual coding following the search to identify reports that met the criteria for analysis.

Only reports involving lithium and lamotrigine were analyzed as there were few search results involving valproic acid (1 on Erowid, 15 on Reddit), carbamazepine (8 on Reddit), and oxcarbazepine (9 on Reddit). Ultimately, 96 total reports involving lithium or lamotrigine were identified and met the criteria to be analyzed. An additional 4 reports involved both lithium and lamotrigine and a concurrent psychedelic-these are reported separately.

Verbatim text from all experience reports is included in the online Supplementary Materials.

\section{Lithium reports}

There were a total of 62 analyzed lithium reports across all 3 sites ( Fig. 1). Erowid had 16 qualifying lithium experience reports (15 [93.8\%] with LSD and 1 [6.2\%] with psilocybin mushrooms). The Shroomery contained 2 lithium trip reports ( 1 with LSD and the other with psilocybin mushrooms). Reddit had 44 qualifying lithium reports (39 with LSD [88.6\%], 4 [9.1\%] with psilocybin mushrooms, and 1 [2.3\%] with DMT).

Of available experience reports from Erowid, the Shroomery, and Reddit involving lithium plus a psychedelic, 29 of 62 (46.8\%) reports were seizures, and an additional 11 of 62 (17.7\%) were bad trips. An additional 12 of 62 (19.4\%) reports were described as intensified. Only 5 of 62 (8.1\%) reports were described as neutral, with lithium not described to affect the psychedelic experience.

Of the 55 reports of lithium plus LSD, 27 (49.1\%) were seizures. Of the 6 reports of lithium plus psilocybin, 2 (33.3\%) were seizures. The sole report of lithium plus DMT did not involve seizures. Removing the 18 lithium reports that contained another psychoactive drug (most commonly serotonin reuptake inhibitors [ $n=5]$ ), $54.5 \%$ of reports still involved seizures.

Notably, $39 \%$ of reports involving lithium and a psychedelic included mention of emergency medical treatment either in the form of an ambulance being called or ending up in a hospital.

Experience reports involving an additional class of more rarely used psychedelics, the $5-\mathrm{HT}_{2 \mathrm{~A}}$ agonist drugs $2 \mathrm{C}-\mathrm{B}, 2 \mathrm{C}-\mathrm{E}$, and $2 \mathrm{C}-\mathrm{I}$,

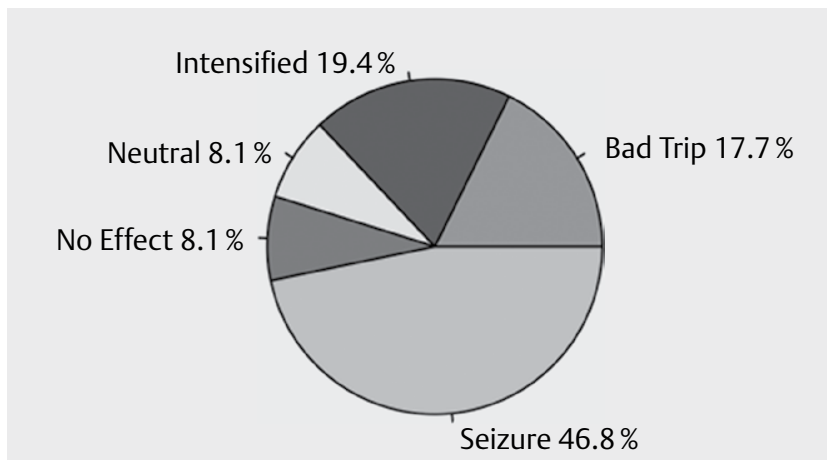

Fig. 1 Effects of taking a classic psychedelic in combination with lithium $(n=62)$. The figure shows the percent distribution of the described psychedelic experiences from websites that archive personal reports of psychedelic use. Categories are mutually exclusive. 


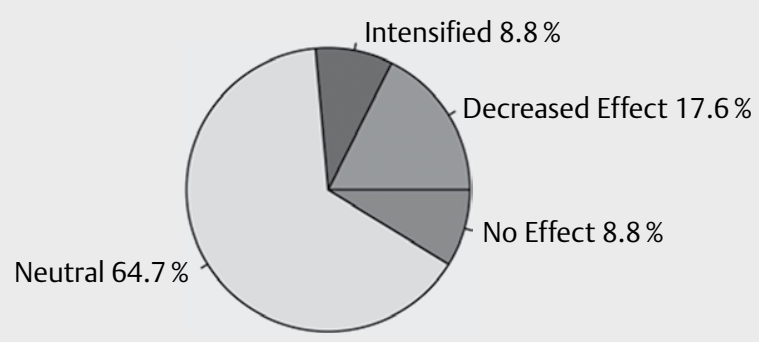

- Fig. 2 Effects of taking a classic psychedelic in combination with lamotrigine $(n=34)$. The figure shows the percent distribution of the described psychedelic experiences from websites that archive personal reports of psychedelic use. Categories are mutually exclusive.

were not included in the main analysis due to the low number of reports (2 Erowid reports for $2 \mathrm{C}$-I with concurrent lithium, 1 Erowid report for $2 \mathrm{C}$-C with concurrent lithium). However, it is noteworthy that both reports involving $2 \mathrm{C}-\mathrm{I}$ use and concurrent lithium involved seizures, and the report of the $2 \mathrm{C}-\mathrm{C}$ plus lithium report was described as intensified.

\section{Lamotrigine reports}

There were a total of 34 analyzed reports of lamotrigine combined with a psychedelic across the 3 sites ( $\triangleright$ Fig. 2). Erowid had 6 reports (3 with LSD, 2 with psilocybin mushrooms, and 1 with smoked DMT). The Shroomery had a single report (psilocybin mushrooms). Reddit had 27 qualifying lamotrigine (or Lamictal) reports (19 [55.9\%] with LSD, 7 [20.6\%] with psilocybin mushrooms, and 1 [2.9\%] with DMT).

In the majority of lamotrigine reports, authors did not clearly express a view that lamotrigine impacted the experience of the psychedelic one way or another. In 22 of 34 (64.7\%) lamotrigine reports, the drug was not reported to have impacted the psychedelic experience. In 6 of 34 (17.6\%) lamotrigine reports, the psychedelic effect was decreased, and in 3 of 34 (8.8\%) reports, there was no psychedelic effect at all. No reports were described as bad trips. The remaining 3 of 34 (8.8\%) reports were described as intensified without an overall negative valence.

Removing the 12 lamotrigine reports that included another psychoactive drug apart from a psychedelic did not substantially change results-in $73.1 \%$ of reports, lamotrigine was not described as affecting the psychedelic experience.

\section{Lithium plus lamotrigine reports}

Excluded from the above analysis were 4 reports that included both lithium and lamotrigine with a psychedelic. One of 4 (25\%) of these reports involved a seizure (though this person also took the MAOI Syrian Rue), and the remaining 3 were described as intensified.

\section{Discussion}

There is a paucity of data about the concurrent use of psychedelic substances with commonly prescribed psychiatric medications, which is concerning given increasing public attention to psilocy- bin-containing mushrooms and the proliferation of enthusiastic and uncritical media reports extolling the benefits of psychedelics such as psilocybin for the treatment of depression and other psychiatric conditions. It is increasingly likely that patients taking conventional psychiatric medications may attempt to treat themselves with psychedelics.

In this study, we collected psychedelic experience reports from 3 large online sources where individuals share such reports (Erowid.org, Shroomery.org, and relevant subreddits from Reddit.com). This study focused on first- or second-person accounts of a psychedelic used in combinations with the commonly prescribed mood stabilizers, lithium or lamotrigine.

\section{Seizures were frequently associated with lithium}

The most notable finding of this study is that $47 \%$ of reports of the use of a classic psychedelic by individuals using lithium involved seizures. This was more frequently reported with LSD but also occurred with psilocybin. As mentioned above, it is also notable that 2 of 3 reports involving a $2 \mathrm{C}$ drug and lithium involved seizures (though these were not included in the main analysis).

\section{Affectively negative experiences ("bad trips") were more common with lithium than lamotrigine}

In addition to reports involving seizures, lithium reports were more likely than lamotrigine reports to be categorized as bad trips ( $18 \%$ versus $0 \%$, respectively).

If seizure reports are excluded (because the categories were mutually exclusive), then 11 of 33 (33\%) non-seizure lithium reports were classified as bad trips.

Below are illustrative examples of verbatim experience reports of lithium and LSD that were classified as non-seizure bad trips:

“Pro Tip: DON'T FUCK WITH THIS. Speaking from experience, Lithium can cause HIGHLY UNPREDICTABLE reactions to dosing. Sometimes 3150 ug hits do nothing or, in my case, 2 hits had me wandering around $[. .$.$] all night with no shoes on in full ego death,$ mumbling to myself like a homeless guy, wandering into traffic, etc. I was fine, but it was SUPER STUPID in hindsight. As such, I no longer take lithium." 1

"I took Isd while on $900 \mathrm{mg}$ of lithium and experienced the worst, most horrific trip of my life and haven't touched Isd since. I had only taken 1 tab but the effects were akin to having doses 5 + tabs. I completely lost all touch with reality, everything I saw was drenched in blood, and I felt my throat closing up and felt like I was on fire-like my skin was burning. I somehow still managed to take a Seroquel despite having lost all touch with reality which brought me out of it after an hour or so I'm guessing (completely lost touch with time) and once I stopped hallucinating I was unable to speak for several hours."2 


\section{Caveats and clinical implications}

The online reports used here are "low quality evidence" [23, 24] due to lack of standardization, selection bias, and lack of clinical verification. Furthermore, it is possible that the same individual posted multiple times on different websites or the same websites with different usernames. In addition, the lithium reports largely did not include information about lithium dose, so we are unable to speculate on dose effects. Despite these caveats, the risk of potentially lethal reactions and the absence of compelling noncausal explanations suggests that the most conservative approach would be to assume a causal relationship-that taking a classic psychedelic (e. g., a non-microdose of LSD or psilocybin) in combination with the therapeutic use of lithium can cause seizures as well as increase the likelihood of otherwise affectively negative experiences.

Thus, we advise individuals taking lithium to avoid taking classic psychedelic drugs. Reports of psychedelic microdoses were not included, thus we are unable to comment on this. It is possible that seizure risk could be mitigated by first tapering and discontinuing lithium, but there is not enough information to make a recommendation.

The benignity of experience reports with lamotrigine does not mean that it is necessarily safe to administer psychedelics to those prescribed it, but the relative lack of signal for increased risk in the analyzed reports could aid future decision-making for clinical trials. For example, a trial of psychedelic treatment for bipolar disorder involving the continuation of mood stabilizers might avoid lithium in favor of lamotrigine due to a lower probability of risk.

\section{Mechanistic conjectures}

There are several possible explanations for seizures in individuals who take psychedelics while using lithium. One possibility is that those who are prescribed lithium may have unique propensities for seizures independent of any risks associated with lithium itself. The current data are limited in that we do not have any medical history information, including seizure risk. People with unipolar depression do have a higher risk of seizure disorder than the general population [25-27], though it is less clear if this is true for bipolar disorder.

A second possible mechanism is that co-administration of lithium and psychedelics could increase systemic concentrations of 1 or both drugs leading to seizures. Though lithium has been found safe to administer to patients with epileptic disorders, elevated lithium levels confer an increased risk for seizures [28]. Classic psychedelics are known to increase cortical excitability [29] and can also, in rare cases, precipitate seizures [30]. However, a metabolic interaction affecting systemic concentrations of either drug is unlikely due to a relative lack of overlap in their metabolic pathways. LSD and psilocybin undergo hepatic metabolism to more water-soluble forms before excretion through urine and other body fluids [31,32]. Lithium, in contrast, is principally renally excreted without undergoing any hepatic alteration.

A third explanation involves changes in local concentrations of lithium and/or psychedelics in the brain. Sartorius et al. also report a case of serotonin syndrome that developed within hours of ECT in a patient who had been treated with lithium, though this did not result in seizures [33]. This raises the question of whether some of the seizures described in these reports occurred in the context of serotonin syndrome. A review of the reports that included seizures found that a substantial portion of cases presented with other signs that may be consistent with serotonin syndrome, including diaphoresis, tachycardia, vomiting, mydriasis, agitation, confusion, and loss of coordination. However, these additional symptoms are more difficult to interpret as they may overlap with the expected effects of psychedelics.

A final and more likely category of explanation would be that concomitant use of lithium and psychedelics results in a synergistic effect on neurotransmission or second messenger effects that increase the likelihood of seizures. The psychoactive effects of psychedelics are mediated primarily by $5-\mathrm{HT}_{2 \mathrm{~A}}$ agonism, and $5-\mathrm{HT}_{2 \mathrm{~A}}$ agonism by psychedelics is generally understood to increase cortical excitability [29,34]. Psychedelics also have an indirect effect of reducing the tonic activity of the locus coeruleus [35-38]. Locus coeruleus activation can be protective against seizures [39-44], suggesting that psychedelic reduction of tonic locus coeruleus activity may represent a potential indirect mechanism by which psychedelic drug administration could reduce the seizure threshold.

\section{Conclusion}

Almost half of online psychedelic experience reports involving lithium and a classic psychedelic (a non-microdose of LSD or psilocybin) involved seizures $(47 \%$ ) and $18 \%$ involved otherwise negative experiences. Furthermore, $39 \%$ of these reports involved emergency medical treatment. In contrast, lamotrigine was not judged to have impacted the experience of the psychedelic drug in the majority of lamotrigine plus psychedelic experiences (64.7\%). Removing all reports that mentioned concurrent use of other psychoactive drugs (most commonly serotonin reuptake inhibitors) did not alter these general findings. Although we acknowledge the substantial limitations of data based on anonymous online psychedelic experience reports, we believe that at present, lithium should be considered a contraindication to psychedelic use.

\section{Acknowledgments}

We thank Alex Cook for providing experience report data that were accessed through the Erowid API.

\section{Funding}

The Center for Psychedelic and Consciousness Research is funded by philanthropic support from the Steven and Alexandra Cohen Foundation, as well as Tim Ferriss, Matt Mullenweg, Blake Mycoskie, and Craig Nerenberg. NG is also funded by NIDA T32DA07209. The funders had no input on the design, execution, or interpretation of this report.

\section{Conflict of Interest}

RRG is on the board of directors of the Heffter Research Institute. SMN serves on a Data Safety and Monitoring Board for Maryland Oncology Hematology. EE and FE are employed by Erowid Center, the non-profit that operates Erowid.org, one of the data sources. 


\section{References}

[1] Davis AK, Barrett FS, May DG et al. Effects of psilocybin-assisted therapy on major depressive disorder: A randomized clinical trial. JAMA Psychiatry 2021; 78: 481-489

[2] Carhart-Harris RL, Bolstridge M, Rucker J et al. Psilocybin with psychological support for treatment-resistant depression: An open-label feasibility study. Lancet Psychiatry 2016; 3: 619-627

[3] Griffiths RR, Johnson MW, Carducci MA et al. Psilocybin produces substantial and sustained decreases in depression and anxiety in patients with life-threatening cancer: A randomized double-blind trial. J Psychopharmacol 2016; 30: 1181-1197

[4] Ross S, Bossis A, Guss J et al. Rapid and sustained symptom reduction following psilocybin treatment for anxiety and depression in patients with life-threatening cancer: a randomized controlled trial. J Psychopharmacol 2016; 30: 1165-1180

[5] Johnson MW, Garcia-Romeu A, Cosimano MP et al. Pilot study of the 5-HT2AR agonist psilocybin in the treatment of tobacco addiction. J Psychopharmacol 2014; 28: 983-992

[6] Bogenschutz MP, Forcehimes AA, Pommy JA et al. Psilocybin-assisted treatment for alcohol dependence: a proof-of-concept study. J Psychopharmacol 2015; 29: 289-299

[7] Schindler EAD, Gottschalk CH, Weil M] et al. Indoleamine hallucinogens in cluster headache: Results of the clusterbusters medication use survey. J Psychoact Drugs 2015; 47: 372-381

[8] Schindler EA, Sewell RA, Gottschalk CH et al. Exploratory controlled study of the migraine-suppressing effects of psilocybin. Neurotherapeutics 2021; 18: 534-543

[9] Flanagan TW, Nichols CD. Psychedelics as anti-inflammatory agents. Int Rev Psychiatry 2018; 30: 363-375

[10] Vieta E, Berk M, Schulze TG et al. Bipolar disorders. Nat Rev Dis Primers 2018; 4: 1-16

[11] Goodwin GM, Haddad PM, Ferrier IN et al. Evidence-based guidelines for treating bipolar disorder: Revised third edition recommendations from the British Association for Psychopharmacology. J Psychopharmacol 2016; 30: 495-553

[12] Geddes JR, Burgess S, Hawton K et al. Long-term lithium therapy for bipolar disorder: Systematic review and meta-analysis of randomized controlled trials. Am J Psychiat 2004; 161: 217-222

[13] McKnight RF, Chesney E, Amit BH et al. Lithium for acute mania. Cochrane Db Syst Rev 2019; 6

[14] Johnson MW, Richards WA, Griffiths RR. Human hallucinogen research: guidelines for safety. J Psychopharmacol 2008; 22: 603-620

[15] Strassman RJ. Adverse reactions to psychedelic drugs. A review of the literature. J Nerv Ment Dis 1984; 172: 577-595

[16] Bonson KR, Murphy DL. Alterations in responses to LSD in humans associated with chronic administration of tricyclic antidepressants, monoamine oxidase inhibitors or lithium. Behavioural Brain Res 1995 73: 229-233

[17] Vollenweider FX, Vollenweider-Scherpenhuyzen MFI, Bäbler A et al. Psilocybin induces schizophrenia-like psychosis in humans via a serotonin-2 agonist action: Neuroreport 1998; 9: 3897-3902. doi:10.1097/00001756-199812010-00024

[18] R Core Team. R: A language and environment for statistical computing.Vienna, Austria: R Foundation for Statistical Computing;; 2020; Im Internet: https://www.R-project.org/

[19] Wickham H. rvest: easily harvest (scrape) web pages. 2020; Im Internet: https://CRAN.R-project.org/package = rvest

[20] Harrison J. RSelenium: R bindings for “Selenium WebDriver”. 2020; Im Internet: https://CRAN.R-project.org/package = RSelenium

[21] Van Rossum G, Drake FL Jr. Python reference manual. Centrum voor Wiskunde en Informatica Amsterdam. 1995;
[22] Boe B. PRAW: the python Reddit API wrapper. 2016; Im Internet: https://praw.readthedocs.org/en/v3.0.0/

[23] Guyatt GH, Oxman AD, Kunz R et al. What is "quality of evidence" and why is it important to clinicians? BMJ 2008; 336: 995-998

[24] Atkins D, Eccles M, Flottorp S et al. Systems for grading the quality of evidence and the strength of recommendations I: critical appraisal of existing approaches The GRADE working group. BMC Health Serv Res 2004; 4: 1-7

[25] Hesdorffer DC, Hauser WA, Annegers JF et al. Major depression is a risk factor for seizures in older adults. Ann Neurol 2000; 47: 246-249

[26] Hesdorffer DC, Hauser WA, Olafsson E et al. Depression and suicide attempt as risk factors for incident unprovoked seizures. Ann Neurol 2006; 59: 35-41

[27] Forsgren L, Nyström L. An incident case-referent study of epileptic seizures in adults. Epilepsy Res 1990; 6: 66-81

[28] Wharton RN. Grand mal seizures with lithium treatment. Am J Psychiat 1969; 125: 1446-1446

[29] Nichols DE. Psychedelics. Pharmacol Rev 2016; 68: 264-355

[30] Fisher DD, Ungerleider JT. Grand mal seizures following ingestion of LSD. Calif Med 1967; 106: 210-211

[31] Libânio Osório Marta RF. Metabolism of lysergic acid diethylamide (LSD): an update. Drug Metab Rev 2019; 51: 378-387

[32] Passie T, Seifert ], Schneider U et al. The pharmacology of psilocybin. Addict Biol 2002; 7: 357-364

[33] Sartorius A, Wolf ], Henn FA. Lithium and ECT-concurrent use still demands attention: three case reports. World J Biol Psychiatry 2005; 6: 121-124

[34] Aghajanian GK, Marek G]. Serotonin induces excitatory postsynaptic potentials in apical dendrites of neocortical pyramidal cells. Neuropharmacology 1997; 36: 589-599

[35] Aghajanian GK. Mescaline and LSD facilitate the activation of locus coeruleus neurons by peripheral stimuli. Brain Res 1980; 186: 492-498

[36] Rasmussen K, Glennon RA, Aghajanian GK. Phenethylamine hallucinogens in the locus coeruleus: potency of action correlates with rank order of 5-HT2 binding affinity. Eur ] Pharmacol 1986; 132: 79-82

[37] Rasmussen K, Aghajanian GK. Effect of hallucinogens on spontaneous and sensory-evoked locus coeruleus unit activity in the rat: Reversal by selective 5-HT2 antagonists. Brain Res 1986; 385: 395-400

[38] Chiang C, Aston-Jones G. A 5-hydroxytryptamine2 agonist augments gamma-aminobutyric acid and excitatory amino acid inputs to noradrenergic locus coeruleus neurons. Neuroscience 1993; 54 : 409-420

[39] Mishra PK, Burger RL, Bettendorf AF et al. Role of norepinephrine in forebrain and brainstem seizures: chemical lesioning of locus ceruleus with DSP4. Exp Neurol 1994; 125: 58-64

[40] Jimenez-Rivera C, Voltura A, Weiss GK. Effect of locus ceruleus stimulation on the development of kindled seizures. Exp Neurol 1987; 95: $13-20$

[41] Giorgi FS, Mauceli G, Blandini F et al. Locus coeruleus and neuronal plasticity in a model of focal limbic epilepsy. Epilepsia 2006; 47: 21-25

[42] Giorgi FS, Blandini F, Cantafora E et al. Activation of brain metabolism and fos during limbic seizures: the role of locus coeruleus. Neurobiol Dis 2008; 30: 388-399

[43] Fornai F, Ruffoli R, Giorgi FS et al. The role of locus coeruleus in the antiepileptic activity induced by vagus nerve stimulation. Eur J Neurosci 2011; 33: 2169-2178

[44] Jerlicz M, Kostowski W, Bidziński A. Audiogenic seizures in rats: relation to noradrenergic neurons of the locus coeruleus. Acta Physiol Pol 1978; 29: 409-412 